\title{
Cost-effectiveness and long-term outcomes of liver transplantation using hepatitis B core antibody-positive grafts with hepatitis B immunoglobulin prophylaxis in Korea
}

Kyeong Deok Kim ${ }^{1, *}$, Ji Eun Lee ${ }^{2, *}$, Jong Man Kim¹, Okjoo Lee', Na Young Hwang ${ }^{3}$, Jinsoo Rhu', Gyu-Seong Choi', Kyunga Kim ${ }^{3}$, and Jae-Won Joh

'Department of Surgery, Samsung Medical Center, Sungkyunkwan University School of Medicine, Seoul; ${ }^{2}$ Department of Radiology, Soonchunhyang University Bucheon Hospital, Soonchunhyang University College of Medicine, Bucheon; ${ }^{3}$ Statistics and Data Center, Research Institute for Future Medicine, Samsung Medical Center, Seoul, Korea

Graphical Abstract

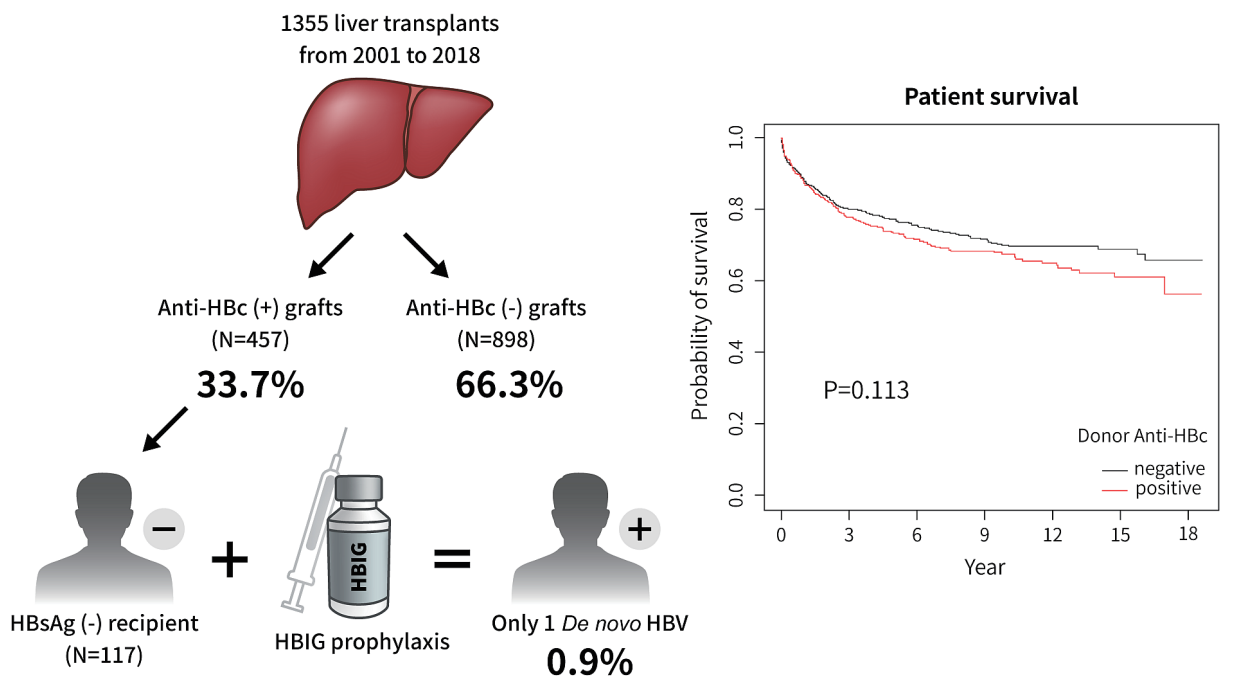

\section{Abbreviations:}

Anti-HBc, hepatitis B core antibody; anti-HBs, hepatitis B surface antibody; BMI, body mass index; DNH, de novo hepatitis B virus infection; HBIG, hepatitis $B$ immunoglobulin; $\mathrm{HBs} A g$, hepatitis B surface antigen; $\mathrm{HBV}$, hepatitis B virus; $\mathrm{HCC}$ hepatocellular carcinoma; HCV, hepatitis C virus; ICU, intensive care unit; LT, liver transplantation; MC, Milan criteria; MELD, model for end-stage liver disease; NAs, nucleos $(t)$ ide analogues

*Kyeong Deok Kim and Ji Eun Lee contributed equally to this study as cofirst authors.
Corresponding author : Jong Man Kim

Department of Surgery, Samsung Medical Center, Sungkyunkwan University School of Medicine, 81 Irwon-ro, Gangnam-gu, Seoul 06351 Korea

Tel: +82-2-3410-1719, Fax: +82-2-3410-0040

E-mail:yjongman21@gmail.com

https://orcid.org/0000-0002-1903-8354

\section{Kyunga Kim}

Statistics and Data Center, Research Institute for Future Medicine Samsung Medical Center, 81 Irwon-ro, Gangnam-gu, Seoul 06351, Korea Tel: +82-2-3410-6745, Fax: +82-2-3410-6745

E-mail: kyunga.j.kim@samsung.com https://orcid.org/0000-0002-0865-2236 
Background/Aims: Hepatitis B core antibody (anti-HBc)-positive donors are used as an extended donor pool, and current guidelines recommend the usage of nucleos(t)ide analogues (NAs) as prophylaxis for preventing de novo hepatitis B virus infection (DNH). We analyzed the long-term outcomes of a large cohort of liver transplantation (LT) patients receiving anti-HBc-positive grafts and evaluated the risk of DNH when hepatitis B immunoglobulin (HBIG) monotherapy was used as prophylaxis. We also compared the cost-effectiveness of HBIG and NAs.

Methods: We retrospectively reviewed 457 patients with anti-HBc-positive grafts and 898 patients with anti-HBcnegative grafts who underwent LT between January 2001 and December 2018. We compared recipient characteristics according to the anti-HBc status of the donor, and compared the costs of using NAs for the rest of the patient's life and using HBIG to maintain hepatitis B surface antibody titers above $200 \mathrm{IU} / \mathrm{L}$.

Results: The 1-, 5-, and 10-year patient survival rates were $87.7 \%, 73.5 \%$, and $67.7 \%$, respectively, in patients with antiHBc-positive grafts, and $88.5 \%, 77.4 \%$, and $70.3 \%$, respectively, in patients with anti-HBc-negative grafts $(P=0.113)$. Among 457 recipients with anti-HBc-positive grafts, 117 (25.6\%) were non-HBV recipients. The overall incidence of DNH was $0.9 \%$. When using HBIG under insurance coverage, the cumulative cost was lower compared with using NA continuously without insurance coverage in Korea.

Conclusions: Anti-HBc-positive grafts alone do not affect patient survival or graft survival. HBIG monoprophylaxis has good outcomes for preventing $\mathrm{DNH}$, and the patient's long-term cost burden is low in Korea because of the national insurance system in this cohort. (Clin Mol Hepatol 2021;27:603-615)

Keywords: Liver transplantation; Hepatitis B virus; Hepatitis B core antibody; De novo hepatitis B virus; Hepatitis B immunoglobulin

\section{Study Highlights}

Using anti-HBc-positive grafts does not impact long-term outcomes after LT. The risk of DNH infection is rare when using hepatitis B core-positive liver grafts with HBIG monoprophylaxis, and the patient's long-term cost burden can be low in Korea considering the insurance system in this cohort.

\section{INTRODUCTION}

De novo hepatitis B virus (HBV) infections (DNH) have been recognized in recipients negative for hepatitis $B$ surface antigen (HBsAg) after liver transplantation (LT) from donors positive for hepatitis B core antibody (anti-HBC) since the early 1990s. ${ }^{1}$ The incidence of DNH was high in recipients who received anti-HBcpositive and HBsAg-negative grafts, because HBV persists in liver grafts even after recovery from previous infection. ${ }^{2}$ There were no prophylaxis options in the 1990s; therefore, the occurrence rates of DNH in recipients were high (43-94\%) and anti-HBc-positive grafts were regarded as inadequate for transplantation. ${ }^{3,4}$ However, HBV infection is endemic in many countries in Asia, and about $65 \%$ of the adult population is anti-HBc-positive in the Republic of Korea. ${ }^{5}$ Thus, many transplant centers have used anti-HBc-positive donors with prophylaxis as an extended donor pool to overcome organ shortages. Prophylaxis strategies are diverse among transplant centers, which use either nucleos(t)ide analogues
(NAs), hepatitis B immunoglobulin (HBIG), or both.

Current international guidelines recommend the use of NAs such as lamivudine as prophylaxis for preventing DNH, while HBIG is not recommended. ${ }^{6,7}$ However, most previous studies of HBIG monotherapy were limited by small sample sizes and short-term follow-up periods. ${ }^{8}$ In addition, the occurrence rates of DNH varied widely from $0 \%$ to $58 \%$ when HBIG alone was used as prophylaxis, and HBIG usage also varied between centers. ${ }^{9-13}$

Thus, in this study we analyzed the long-term outcomes of a large cohort of LT patients receiving anti-HBc-positive grafts at a single center, and evaluated the risks and outcomes of DNH when HBIG monotherapy was used as prophylaxis. In addition, we compared the cost-effectiveness of HBIG and NAs.

\section{MATERIALS AND METHODS}

This study was approved by the Samsung Medical Center Insti- 
tutional Review Board (IRB No. 2020-11-164). The need for consent from participants was exempted by the IRB.

\section{Patients}

We retrospectively reviewed all patients who underwent LT at Samsung Medical Center in Seoul, Korea, between January 2001 and December 2018. Figure 1 shows a flow chart summarizing the patient selection process. We excluded recipients who received livers of deceased donors from other hospitals because their viral statuses were not consistently documented, as well as recipients or donors with no recorded pre-op anti-HBc status. We also excluded recipients younger than 18 and recipients undergoing re-transplantation. In the end, 1,355 patients were enrolled in this study.

\section{Immunosuppression}

All recipients received induction therapy with basiliximab. Maintenance therapy was primarily achieved using a triple immunosup- pressive regimen consisting of calcineurin inhibitors, mycophenolate mofetil, and corticosteroids. Corticosteroids were discontinued 3 months after LT except for patients who underwent LT for autoimmune liver disease or had a history of rejection.

\section{HBV prophylaxis}

HBV recipients received combination treatments with $\mathrm{HBIG}$ and NAs, which were used before LT. For HBV patients who were either hepatitis B e-antigen positive or HBV DNA positive before transplantation, $\mathrm{HBIG}(20,000 \mathrm{IU})$ was administered first 10 times after surgery, and then HBIG (10,000 IU) was administered. All $\mathrm{HBV}$ recipients, regardless of the anti- $\mathrm{HBC}$ status of the donor and hepatitis B surface antibody (anti-HBs) titer of the recipient, received HBIG (10,000 IU) at each outpatient visit (2-3 months) and took NAs for the rest of their lives to prevent HBV recurrence. Non-HBV recipients were given HBIG monotherapy as HBV prophylaxis when receiving anti-HBc-positive grafts. Generally, HBIG $(10,000 \mathrm{IU})$ was administered during the anhepatic phase and six times after LT. Anti-HBs titers were routinely monitored at each

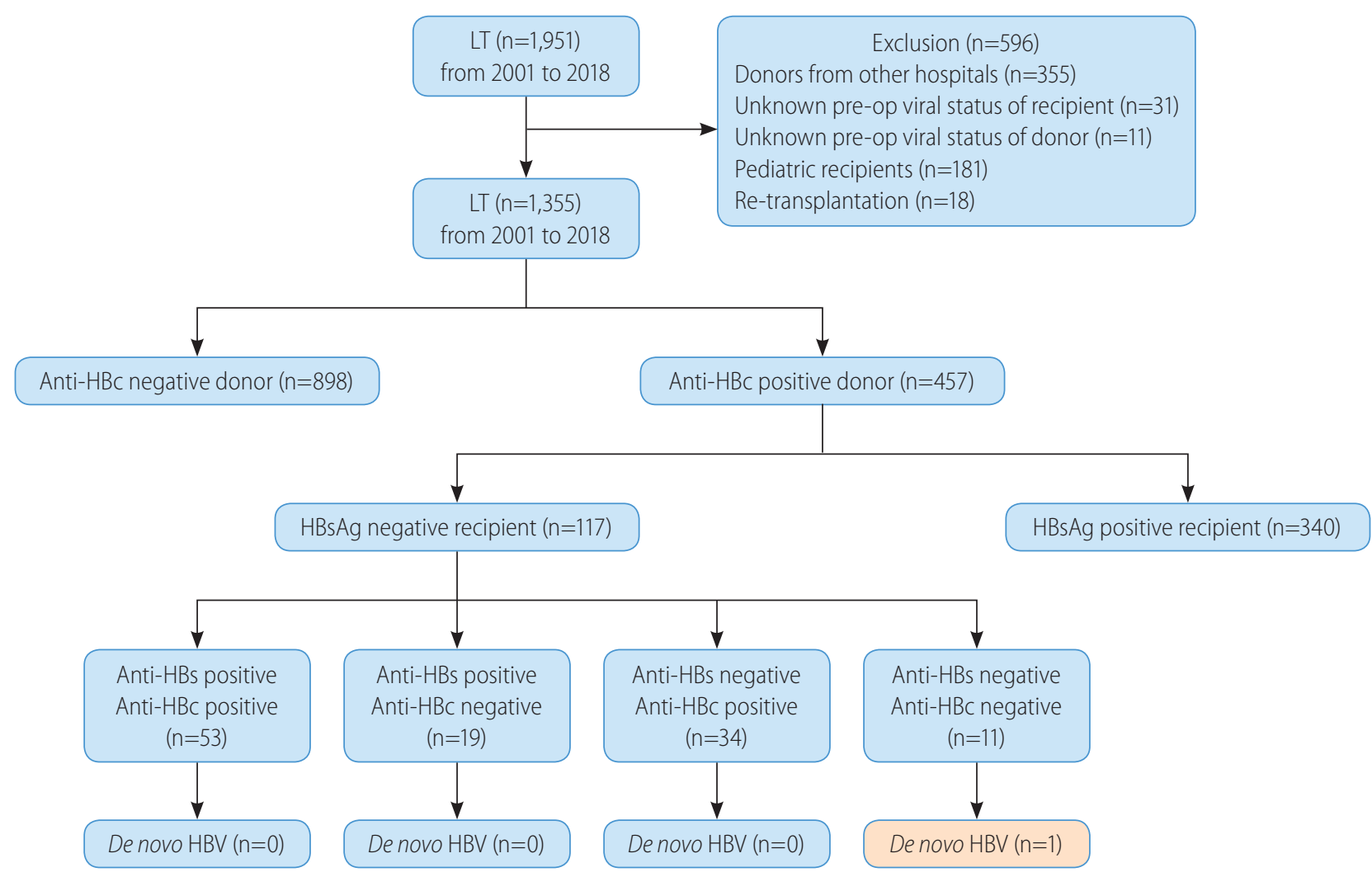

Figure 1. Flow chart of the patient selection process and allocation of anti-HBc-positive grafts. LT, liver transplantation; anti-HBC, hepatitis B core antibody; HBsAg, hepatitis B surface antigen; anti-HBs, hepatitis B surface antibody; HBV, hepatitis B virus. 
outpatient visit (2-3 months) to maintain titers above $200 \mathrm{IU} / \mathrm{L}$. HBIG is administered when the anti-HBs titer is below $200 \mathrm{IU} / \mathrm{L}$, regardless of the recipient's HBV serological status before transplantation. However, non-HBV recipients who received anti-HBCnegative grafts did not require surveillance for HBV.

\section{Clinical parameters}

We retrospectively analyzed donor and recipient data. Donors were compared based on age, sex, body mass index (BMI) $\left(\mathrm{kg} / \mathrm{m}^{2}\right)$, type of donor, warm and cold ischemic time, and day 0 graft biopsy according to anti-HBC status. The recipient data collected were age, sex, BMI, model for end-stage liver disease (MELD) score, presence of HBV, hepatitis C virus (HCV), and hepatocellular carcinoma (HCC) according to the Milan criteria (MC), operation time, postoperative intensive care unit (ICU) stay period, hospitalization period, complication rate, severe complication rate, and mortality within 30 days. The MC was defined as single HCC $\leq 5 \mathrm{~cm}$ or up to $3 \mathrm{HCCs}$ none exceeding $3 \mathrm{~cm}$, without vascular invasion. ${ }^{14}$ Severe complication was defined as Clavien-Dindo classification 3a or higher. DNH was defined as the development of positive HBsAg and/or detectable HBV DNA in a non-HBV recipient.

\section{Statistical analysis}

Continuous variables are presented as mean \pm standard deviation and were compared using the Mann-Whitney U test. Categorical variables are presented as numbers with percentages and were compared by chi-square tests. Patient survival was measured from the time of transplantation to death from any cause. Graft loss was defined as recipient death, being listed for re-transplantation, or undergoing re-transplantation. Patient, graft, and disease-free survival were estimated using the Kaplan-Meier method and compared using log-rank tests. Cox proportional hazards model analysis was used to predict patient and graft survival. Multivariable analysis was performed using factors that were significant clinically or in univariable analysis $(P<0.05)$. Yearly and cumulative costs for HBIG were estimated by the simple moving average method using the function 'sma' in the R package 'smooth'. All tests were two-tailed and statistical significance was defined as $P<0.05$. All statistical analyses were done using the software SPSS (version 25; IBM, Armonk, NY, USA) and R (version 3.5.2; R Foundation for Statistical Computing, Vienna, Austria).

\section{RESULTS}

\section{Donor and recipient clinical characteristics by anti- $\mathrm{HBC}$ status of donor}

Four hundred and fifty-seven patients $(33.7 \%, 457 / 1,355)$ were included in the anti-HBc-positive group and 898 patients $(66.3 \%$, $898 / 1,355)$ were included in the anti-HBc-negative group. The median follow-up time for the cohort was 5.7 years (2.0-10.5): 6.4 years $(2.0-11.3)$ for the anti-HBC-positive group and 5.5 years (2.0-10.1) for the anti-HBC-negative group. The comparison of donor and recipient characteristics according to donor anti-HBC status is shown in Table 1. Donor age and BMI were higher in the anti-HBc-positive group ( $P<0.001$ and $P=0.010$, respectively). The proportions of males and living donors were higher in the antiHBC-negative group $(P<0.001$ and $P=0.005$, respectively). Recipient age was higher in the anti-HBC-negative group $(P=0.027)$. The proportion of male recipients was higher in the anti-HBc-negative group $(P=0.001)$. The proportion of transplant indications was significantly different between the two groups $(P=0.039)$; acute, acute on chronic, and cirrhosis were $5.3 \%, 8.3 \%$, and $86.4 \%$ in the anti-HBc-positive group, respectively, and $4.1 \%, 5.1 \%$, and $90.8 \%$ in the anti-HBc-negative group, respectively. MELD score was higher in the anti-HBC-positive group $(P=0.009)$. The proportion of HBV recipients and macrovesicular and microvesicular fatty change above 10 percent on day $0 \mathrm{graft}$ biopsy were higher in the anti-HBc-positive group ( $P=0.011, P=0.005$, and $P=0.026$, respectively). Hospitalization periods were longer in the anti-HBCpositive group $(P=0.033)$. However, recipient BMI, HCV recipients, HCC patients according to the MC, operation time, warm and cold ischemic time, postoperative ICU stay period, complication rate, and mortality within 30 days did not differ significantly between the two groups.

\section{De novo HBV infection}

Figure 1 shows the allocation of anti-HBc-positive grafts in our cohort. One hundred and seventeen non-HBV recipients $(25.6 \%$, $117 / 457$ ) received anti-HBc-positive grafts; 53 recipients (45.3\%, 53/117) were both anti-HBs- and anti-HBc-positive, 19 recipients $(16.2 \%, 19 / 117)$ were anti-HBs-positive and anti-HBc-negative, 34 recipients $(29.1 \%, 34 / 117)$ were anti-HBs-negative and anti-HBCpositive, and 11 recipients $(9.4 \%, 11 / 117)$ were both anti-HBsand anti-HBc-negative. There was only one DNH out of $11(9.1 \%)$ anti-HBs-negative and anti-HBC-negative recipients who had not 
Table 1. Clinical characteristics of donors and recipients according to donor anti-HBc status

\begin{tabular}{|c|c|c|c|}
\hline & Anti-HBc-positive group $(n=457)$ & Anti-HBc-negative group $(n=898)$ & $P$-value \\
\hline \multicolumn{4}{|l|}{ Donor characteristic } \\
\hline Age (years) & $41.3 \pm 11.9$ & $29.6 \pm 10.3$ & $<0.001$ \\
\hline Sex, male & $260(56.9)$ & $620(69.0)$ & $<0.001$ \\
\hline $\mathrm{BMI}\left(\mathrm{kg} / \mathrm{m}^{2}\right)$ & $23.5 \pm 3.1$ & $23.1 \pm 3.1$ & 0.010 \\
\hline Living donors & $417(91.2)$ & $854(95.1)$ & 0.005 \\
\hline \multicolumn{4}{|l|}{ Recipient characteristic } \\
\hline Age (years) & $51.3 \pm 9.9$ & $52.5 \pm 8.5$ & 0.027 \\
\hline Sex, male & $334(73.1)$ & $726(80.8)$ & 0.001 \\
\hline $\mathrm{BMI}\left(\mathrm{kg} / \mathrm{m}^{2}\right)$ & $24.4 \pm 3.8$ & $24.4 \pm 3.5$ & 0.938 \\
\hline Transplant indication & & & 0.039 \\
\hline Acute & $24(5.3)$ & $37(4.1)$ & \\
\hline Acute on chronic & $38(8.3)$ & $46(5.1)$ & \\
\hline Cirrhosis & $395(86.4)$ & $815(90.8)$ & \\
\hline MELD score & $19.2 \pm 10.5$ & $17.7 \pm 10.1$ & 0.009 \\
\hline HBV infection & $348(76.1)$ & $625(69.6)$ & 0.011 \\
\hline HCV infection & $23(5.0)$ & $44(4.9)$ & 0.915 \\
\hline $\mathrm{HCC}$ & & & 0.815 \\
\hline No HCC & $197(43.1)$ & $371(41.3)$ & \\
\hline Within the Milan criteria & $179(39.2)$ & $361(40.2)$ & \\
\hline Beyond the Milan criteria & $81(17.7)$ & $166(18.5)$ & \\
\hline Warm ischemic time (minutes) & $38.4 \pm 19.4$ & $38.2 \pm 17.6$ & 0.881 \\
\hline Cold ischemic time (minutes) & $95.0 \pm 63.0$ & $97.4 \pm 66.0$ & 0.537 \\
\hline Operation time (minutes) & $552.2 \pm 117.6$ & $553.2 \pm 128.0$ & 0.887 \\
\hline \multicolumn{4}{|l|}{ Day 0 graft biopsy, steatosis } \\
\hline Macrovesicular fatty change $>10 \%$ & $72(15.8)$ & $94(10.5)$ & 0.005 \\
\hline Microvesicular fatty change $>10 \%$ & $124(27.1)$ & $195(21.7)$ & 0.026 \\
\hline Postoperative ICU stay period (days) & $9.2 \pm 11.4$ & $8.2 \pm 7.3$ & 0.105 \\
\hline Hospitalization period (days) & $41.9 \pm 37.7$ & $37.7 \pm 28.6$ & 0.033 \\
\hline Complication rate & $210(46.0)$ & $441(49.1)$ & 0.271 \\
\hline Severe complication within 30 days* & $157(34.4)$ & $303(33.7)$ & 0.822 \\
\hline Recipient mortality within 30 days & $13(2.8)$ & $27(3.0)$ & 0.868 \\
\hline
\end{tabular}

Values are presented as mean \pm standard deviation or number (\%).

Anti-HBC, hepatitis B core antibody; BMI, body mass index; MELD, model for end stage liver disease; HBV, hepatitis B virus; HCV, hepatitis C virus; HCC, hepatocellular carcinoma; ICU, intensive care unit.

*Clavien-Dindo classification $\geq$ grade 3 a.

experienced HBV infection or immunity. The overall incidence of DNH was $0.9 \%$ (1/117). The patient who developed DNH had non-HBV and non-HCV HCC with Budd-Chiari syndrome and underwent LT. Before LT, HCC was treated with radiofrequency ablation and trans-arterial chemoembolization for 5 years. After LT, the patient received HBIG six times before discharge and the anti-
HBs titer was maintained above $200 \mathrm{IU} / \mathrm{L}$ for 6 months. After 6 months, the titer fell to $145 \mathrm{IU} / \mathrm{L}$ and the patient received $\mathrm{HBIG}$ once again, and the titer was maintained above $200 \mathrm{IU} / \mathrm{L}$ for 3 months. At 10 months post-LT follow-up, the anti-HBs titer fell below $200 \mathrm{IU} / \mathrm{L}$ again. However, HBIG administration was not performed for the following 4 months. The patient received HBIG 


\section{CLINCAL And MOLECULAA \\ HEPATOLOGY}

Volume_27 Number_4 October 2021

at every outpatient clinic follow-up afterwards, but the anti-HBs titer remained negative. At 21 months after LT, HBV DNA copies were high, resulting in DNH. After 2 months, HCC recurred in the lung, and the patient expired after 7 months.

\section{Patient and graft survival according to anti-HBC status of donor}

Anti-HBc status of the donor did not affect patient survival. The $1-, 5-$, and 10-year patient survival rates were $87.7 \%, 73.5 \%$, and
$67.7 \%$, respectively, in the anti-HBc-positive group and $88.5 \%$, $77.4 \%$, and $70.3 \%$, respectively, in the anti-HBc-negative group $(P=0.113)$ (Fig. 2A). On the other hand, there were significant differences in graft survival rates according to anti-HBc status. The 1 -, 5-, and 10-year graft survival rates were $86.6 \%, 70.9 \%$, and $64.1 \%$, respectively, in the anti-HBc-positive group and $87.3 \%$, $76.1 \%$, and $68.7 \%$, respectively, in the anti-HBc-negative group $(P=0.046)$ (Fig. 2B). In addition, anti-HBc status of the donor did not affect patient survival in high MELD patients (defined as MELD $\geq 35$ ). In high MELD patients, the 1-, 5-, and 10-year pa-
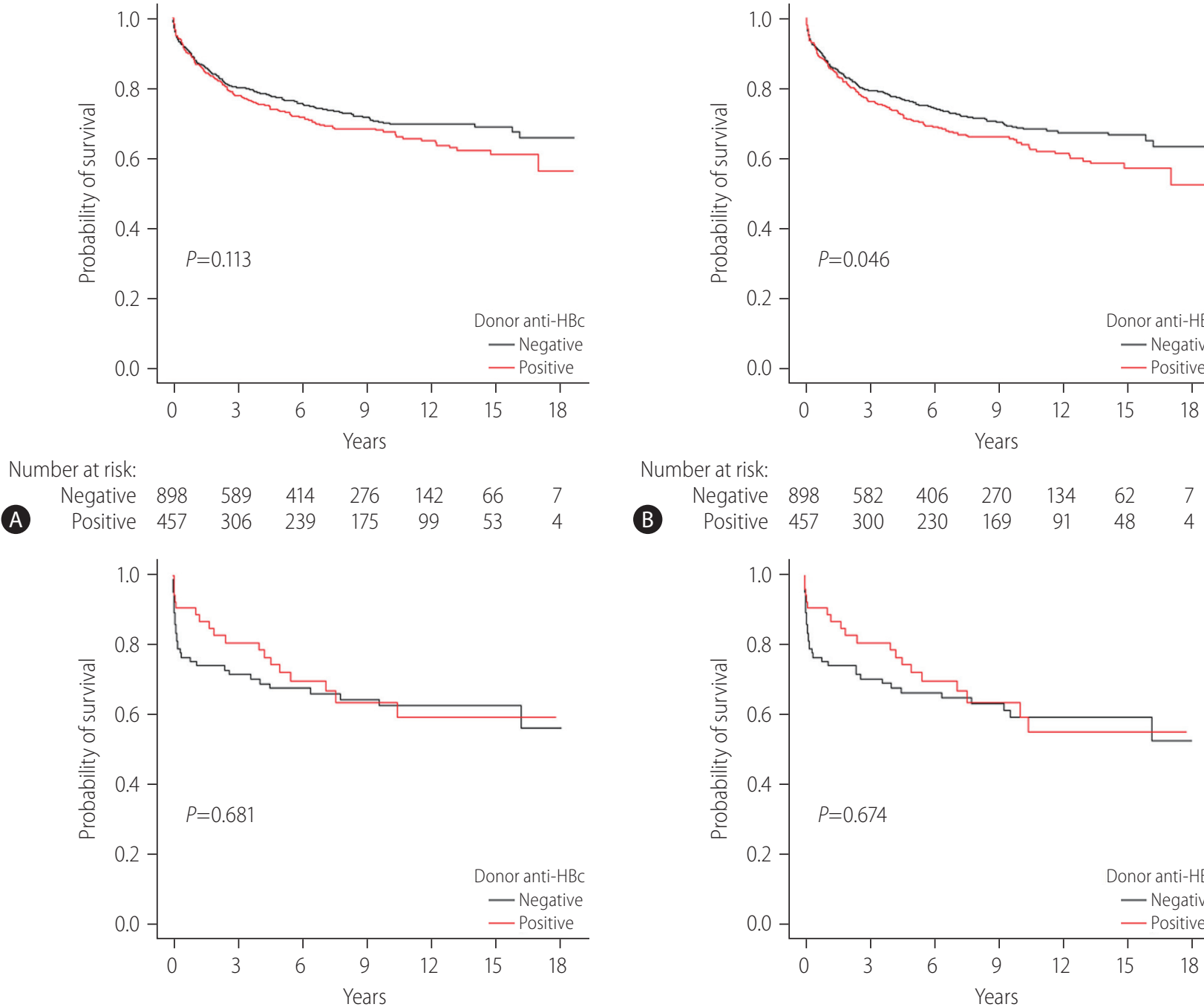

Number at risk:

$\begin{array}{rlllllll}\text { C Negative } & 86 & 56 & 44 & 35 & 23 & 14 & 0 \\ \text { Positive } & 54 & 40 & 28 & 17 & 10 & 7 & 0\end{array}$

Number at risk:

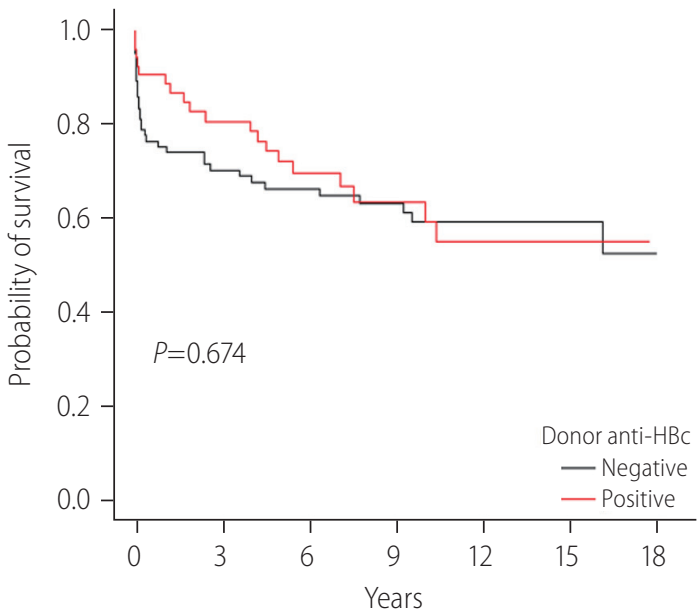

$\begin{array}{cccccccc}\text { Degative } & 86 & 55 & 43 & 34 & 21 & 13 & 0 \\ \text { Dositive } & 54 & 40 & 28 & 17 & 9 & 6 & 0\end{array}$

Figure 2. Patient and graft survival stratified by donor anti-HBC status. (A) Patient survival stratified by donor anti-HBC status. (B) Graft survival stratified by donor anti-HBC status. (C) Patient survival stratified by donor anti-HBC status in high MELD ( $\geq 35$ ) patients. (D) Graft survival stratified by donor anti-HBC status in high MELD ( $\geq 35$ ) patients. Group comparisons were performed using Kaplan-Meier and log-rank tests. Anti HBc, hepatitis B core antibody; MELD, model for end-stage liver disease. 
tient survival rates were similar between the two groups, where the anti-HBc-positive group survival rates were 90.7\%, 72.4\%, and $63.9 \%$, and the anti-HBc-negative group survival rates were
$75.6 \%, 67.8 \%$, and $62.8 \%$, respectively $(P=0.681)$ (Fig. $2 \mathrm{C})$. In high MELD patients, the anti-HBC status of the donor did not influence graft survival either. The 1-, 5-, and 10-year graft survival

Table 2. Causes of graft loss in the whole cohort

\begin{tabular}{|c|c|c|c|}
\hline & Anti-HBc-positive group ( $n=457$ ) & Anti-HBc-negative group $(n=898)$ & Total \\
\hline Total number of graft losses & $156(34.1)$ & $241(26.8)$ & 397 \\
\hline \multicolumn{4}{|l|}{ Liver complication } \\
\hline Vascular complication & $18(11.5)$ & $17(7.1)$ & 35 \\
\hline Biliary complication & $16(10.3)$ & $15(6.2)$ & 31 \\
\hline Rejection & $5(3.2)$ & $15(6.2)$ & 20 \\
\hline Alcoholic cirrhosis & $1(0.6)$ & $2(0.8)$ & 3 \\
\hline Other & $6(3.9)$ & $11(4.6)$ & 17 \\
\hline \multicolumn{4}{|l|}{ Recurrence of original disease } \\
\hline HBV infection & $2(1.3)$ & $0(0.0)$ & 2 \\
\hline HCV infection & $1(0.6)$ & $4(1.7)$ & 5 \\
\hline $\mathrm{PBC} / \mathrm{PSC}$ & $0(0.0)$ & $1(0.4)$ & 1 \\
\hline \multicolumn{4}{|l|}{ Malignancy } \\
\hline Recurrence of original tumor & $42(26.9)$ & $80(33.2)$ & 122 \\
\hline De novo solid tumor & $5(3.2)$ & $7(2.9)$ & 12 \\
\hline Post-transplanted lymphoproliferative disease & $1(0.6)$ & $2(0.8)$ & 3 \\
\hline Patient death from other causes & $59(37.8)$ & $87(36.1)$ & 146 \\
\hline
\end{tabular}

Values are presented as number (\%).

Statistical analysis was not performed to determine the cause of graft loss.

Anti-HBc, hepatitis B core antibody; HBV, hepatitis B virus; HCV, hepatitis c virus; PBC, primary biliary cirrhosis; PSC, primary sclerosing cholangitis.

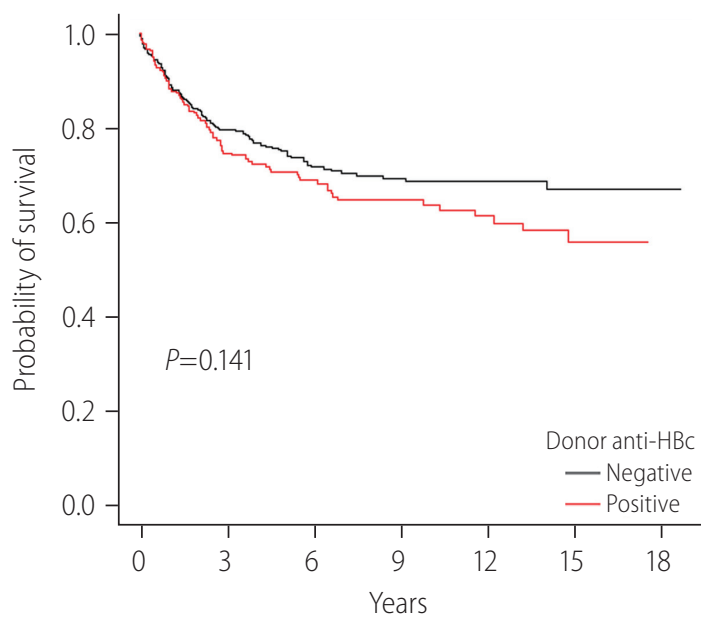

Number at risk:

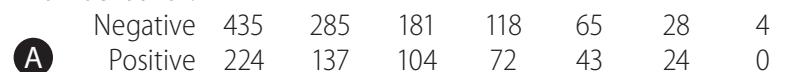

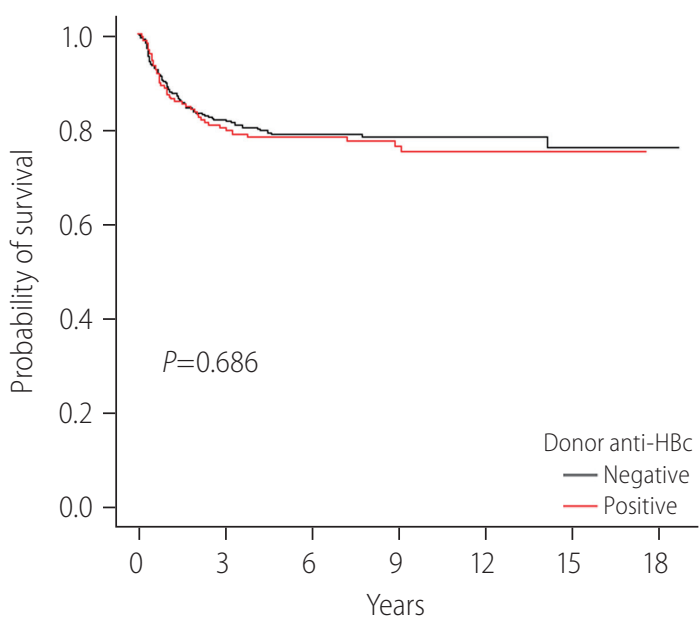

Number at risk:

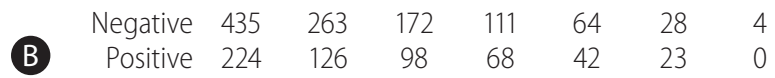

Figure 3. Patient survival and disease-free survival rates in HBV recipients who underwent LT due to HCC stratified by donor anti-HBC status. (A) Patient survival in HBV recipients who underwent LT due to HCC stratified by donor anti-HBC status. (B) Disease-free survival rates in HBV recipients who underwent LT due to HCC stratified by donor anti-HBC status. Group comparisons were performed using Kaplan-Meier and log-rank tests. Anti-HBC, hepatitis B core antibody; HBV, hepatitis B virus; LT, liver transplantation; HCC, hepatocellular carcinoma. 


\section{CLINICAL and MOLECULAR}

rates were as follows: anti-HBc-positive group (90.7\%, 72.4\%, and $59.6 \%)$ and anti-HBc-negative group $(75.6 \%, 66.6 \%$, and $59.6 \%, P=0.674$ ) (Fig. 2D). There were 397 graft losses at the time of analysis: 156 graft losses in the anti-HBc-positive group $(34.1 \%, 156 / 457)$ and 241 graft losses in the anti-HBc-negative group $(26.8 \%, 241 / 898)$ (Table 2$)$. The majority of graft losses were due to recurrence of the original tumor or other causes (infection, cardiovascular events, or trauma). There were no graft losses due to DNH (Table 2).

\section{Outcomes of LT in HBV recipients with HCC according to donor anti-HBc status}

Of 973 HBV recipients, 659 (67.7\%) had concurrent HCC at the time of surgery. Approximately one-third of the patients received anti-HBc-positive grafts (224/659 [34.0\%]) and the rest received anti-HBc-negative grafts (435/659 [66.0\%]). In recipients with HCC, the 1-, 5-, and 10-year patient survival rates were $88.8 \%$, $70.8 \%$, and $63.8 \%$, respectively in those with anti-HBc-positive grafts, and $89.8 \%, 75.1 \%$, and $68.9 \%$, respectively for those

Table 3. Univariable and multivariable analyses of risk factors for patient survival

\begin{tabular}{|c|c|c|c|c|}
\hline \multirow{2}{*}{ Variable } & \multicolumn{2}{|c|}{ Univariable } & \multicolumn{2}{|c|}{ Multivariable } \\
\hline & $\mathrm{HR}(95 \% \mathrm{Cl})$ & $P$-value & HR $(95 \% \mathrm{CI})$ & $P$-value \\
\hline Donor age & $1.008(0.999-1.016)$ & 0.069 & & \\
\hline Male donor & $0.858(0.694-1.059)$ & 0.154 & & \\
\hline Donor BMI & 1.018 (0.987-1.051) & 0.259 & & \\
\hline Living donor & $1.212(0.780-1.883)$ & 0.392 & & \\
\hline Anti-HBc-positive graft & 1.185 (0.961-1.461) & 0.113 & $1.200(0.972-1.481)$ & 0.089 \\
\hline Recipient age & $1.003(0.991-1.015)$ & 0.600 & & \\
\hline Male recipient & 1.207 (0.929-1.568) & 0.158 & & \\
\hline Recipient BMI & $0.970(0.941-1.000)$ & 0.049 & $0.970(0.940-1.000)$ & 0.048 \\
\hline Transplant indication & & 0.163 & & \\
\hline Acute & 1 (reference) & & & \\
\hline Acute on chronic & 1.415 (0.768-2.605) & 0.266 & & \\
\hline Cirrhosis & $0.978(0.592-1.616)$ & 0.931 & & \\
\hline Recipient MELD score & 1.007 (0.997-1.017) & 0.179 & & \\
\hline Recipient HCC & & $<0.001$ & & $<0.001$ \\
\hline No HCC & 1 (reference) & & 1 (reference) & \\
\hline Within the Milan criteria & $1.102(0.870-1.397)$ & 0.420 & 1.255 (0.980-1.608) & 0.089 \\
\hline Beyond the Milan criteria & $1.953(1.502-2.539)$ & $<0.001$ & $2.158(1.640-2.840)$ & $<0.001$ \\
\hline Recipient HBV infection & $0.766(0.614-0.956)$ & 0.018 & $0.700(0.554-0.885)$ & 0.003 \\
\hline Recipient HCV infection & $1.692(1.151-2.487)$ & 0.007 & $1.263(0.817-1.951)$ & 0.294 \\
\hline \multicolumn{5}{|l|}{ Day 0 graft biopsy, steatosis } \\
\hline Macrovesicular fatty change $>10 \%$ & $1.050(0.781-1.412)$ & 0.747 & & \\
\hline Microvesicular fatty change $>10 \%$ & $0.955(0.754-1.210)$ & 0.702 & & \\
\hline Cold ischemic time & $1.000(0.998-1.001)$ & 0.921 & & \\
\hline Warm ischemic time & $0.999(0.993-1.005)$ & 0.627 & & \\
\hline Severe complication within 30 days* & $2.299(1.873-2.823)$ & $<0.001$ & $2.183(1.776-2.683)$ & $<0.001$ \\
\hline Rejection episode & $0.951(0.729-1.242)$ & 0.713 & & \\
\hline
\end{tabular}

$\mathrm{HR}$, hazard ratio; $\mathrm{Cl}$, confidence interval; $\mathrm{BMI}$, body mass index; anti-HBC, hepatitis B core antibody; MELD, model for end stage liver disease; HCC, hepatocellular carcinoma; HBV, hepatitis B virus; HCV, hepatitis c virus.

*Clavien-Dindo classification $\geq$ grade 3 a. 
with anti-HBC-negative grafts ( $P=0.141)$ (Fig. 3A). The risk of HCC recurrence was the same regardless of donor anti-HBC status. The 1-, 5-, and 10-year disease-free survival rates of patients with HCC were $87.3 \%, 78.3 \%$ and $75.1 \%$, respectively, in the antiHBC-positive group and $89.2 \%, 78.8 \%$ and $78.2 \%$, respectively, in the anti-HBC-negative group ( $P=0.686)$ (Fig. 3B).

\section{Univariable and multivariable analyses of risk factors for patient and graft survival}

Anti-HBc-positive grafts were not a significant risk factor asso- ciated with patient survival in both univariable and multivariable analysis ( $P=0.113$ and $P=0.093$, respectively) (Table 3). Although anti-HBc-positive grafts seemed to be a risk factor associated with graft survival in univariable analysis $(P=0.046)$, it was not a significant risk factor in multivariable analysis $(P=0.266)$ (Table 4). In multivariable analysis, recipient BMI and HBV infection were associated with better patient survival $(P=0.048$ and $P=0.003$, respectively). However, $\mathrm{HCC}$ beyond the MC and postoperative severe complication within 30 days were associated with poorer patient survival $(P<0.001$ and $P<0.001$, respectively) (Table 3). Similarly, recipient BMI and HBV infection were found to be asso-

Table 4. Univariable and multivariable analyses of risk factors for graft survival

\begin{tabular}{|c|c|c|c|c|}
\hline \multirow{2}{*}{ Variable } & \multicolumn{2}{|c|}{ Univariable } & \multicolumn{2}{|c|}{ Multivariable } \\
\hline & $\mathrm{HR}(95 \% \mathrm{Cl})$ & $P$-value & HR $(95 \% \mathrm{Cl})$ & $P$-value \\
\hline Donor age & $1.011(1.003-1.019)$ & 0.007 & $1.010(1.002-1.018)$ & 0.016 \\
\hline Male donor & $0.854(0.696-1.047)$ & 0.128 & & \\
\hline Donor BMl & $1.021(0.990-1.052)$ & 0.182 & & \\
\hline Living donor & $1.254(0.816-1.928)$ & 0.302 & & \\
\hline Anti-HBc positive graft & $1.228(1.004-1.502)$ & 0.046 & $1.138(0.906-1.429)$ & 0.266 \\
\hline Recipient age & $0.999(0.988-1.010)$ & 0.820 & & \\
\hline Male recipient & $1.186(0.922-1.524)$ & 0.184 & & \\
\hline Recipient BMI & $0.962(0.934-0.991)$ & 0.011 & $0.964(0.936-0.993)$ & 0.015 \\
\hline Transplant indication & & 0.254 & & \\
\hline Acute & 1 (reference) & & & \\
\hline Acute on chronic & $1.363(0.752-2.473)$ & 0.308 & & \\
\hline Cirrhosis & $0.995(0.611-1.620)$ & 0.170 & & \\
\hline Recipient MELD score & $1.007(0.997-1.016)$ & 0.170 & & \\
\hline Recipient HCC & & $<0.001$ & & $<0.001$ \\
\hline No HCC & 1 (reference) & & 1 (reference) & \\
\hline Within the Milan criteria & $1.103(0.879-1.384)$ & 0.396 & $1.238(0.977-1.568)$ & 0.137 \\
\hline Beyond the Milan criteria & $1.818(1.407-2.349)$ & $<0.001$ & $1.950(1.493-2.547)$ & $<0.001$ \\
\hline Recipient HBV infection & $0.794(0.640-0.985)$ & 0.036 & $0.774(0.617-0.973)$ & 0.028 \\
\hline Recipient HCV infection & $1.571(1.070-2.308)$ & 0.021 & $1.223(0.794-1.883)$ & 0.362 \\
\hline \multicolumn{5}{|l|}{ Day 0 graft biopsy (steatosis) } \\
\hline Macrovesicular fatty change $>10 \%$ & $1.049(0.789-1.395)$ & 0.743 & & \\
\hline Microvesicular fatty change $>10 \%$ & $0.969(0.801-1.259)$ & 0.969 & & \\
\hline Cold ischemic time & 1.000 (0.998-1.001) & 0.881 & & \\
\hline Warm ischemic time & $0.999(0.993-1.005)$ & 0.719 & & \\
\hline Severe complication within 30 days* & $2.410(1.976-2.937)$ & $<0.001$ & $2.323(1.904-2.836)$ & $<0.001$ \\
\hline Rejection episode & $1.038(0.807-1.334)$ & 0.773 & & \\
\hline
\end{tabular}

HR, hazard ratio; $\mathrm{Cl}$, confidence interval; BMI, body mass index; anti-HBC, hepatitis B core antibody; MELD, model for end stage liver disease; HCC, hepatocellular carcinoma; HBV, hepatitis B virus; HCV, hepatitis c virus.

*Clavien-Dindo classification $\geq$ grade 3 a. 


\section{CLINICAL and MOLECULAR}

Volume_27 Number_4 October 2021

ciated with better graft survival in multivariable analysis $(P=0.015$ and $P=0.028$, respectively). However, donor age, HCC beyond the $M C$, and postoperative severe complications within 30 days were associated with poorer graft survival $(P=0.016, P<0.001$, and $P<0.001$, respectively) (Table 4).

\section{Cost analysis between HBIG and nucleotide analogues}

Non-HBV recipients who receive anti-HBc-positive grafts need to take NAs for the rest of their lives or have HBIG administered intermittently to maintain anti-HBs titers above 200 IU/L. In our study, all 117 of the non-HBV recipients were given HBIG monotherapy as HBV prophylaxis when receiving anti-HBc-positive grafts. In addition, $46 \%$ (54/117) of the patients were adminis- tered their last dose of HBIG within 2 years after LT (Fig. 4A). We compared between the hypothetical costs the patients would have paid if they used NAs for the rest of their lives to the actual cost for using HBIG to maintain anti-HBs titers above $200 \mathrm{IU} / \mathrm{L}$. When using HBIG, the cumulative cost was higher compared to using NAs continuously (Fig. 4B). In terms of the cost distribution among HBIG use, 8.5\% (10/117) had lower costs than entecavir, and none had lower costs than lamivudine (Supplementary Fig. 1). However, only the use of HBIG was covered by Korean insurance system until 2017 and the cost to be paid by the patient was reduced to $10 \%$. In this case, when using HBIG, the cost is lower after 9 months compared to using entecavir, and the cost is lower after 44 months compared to using lamivudine (Fig. 4C). Since 2018, the Korean insurance system has covered NAs as well as HBIG. Thus, the cumulative cost of using HBIG became higher
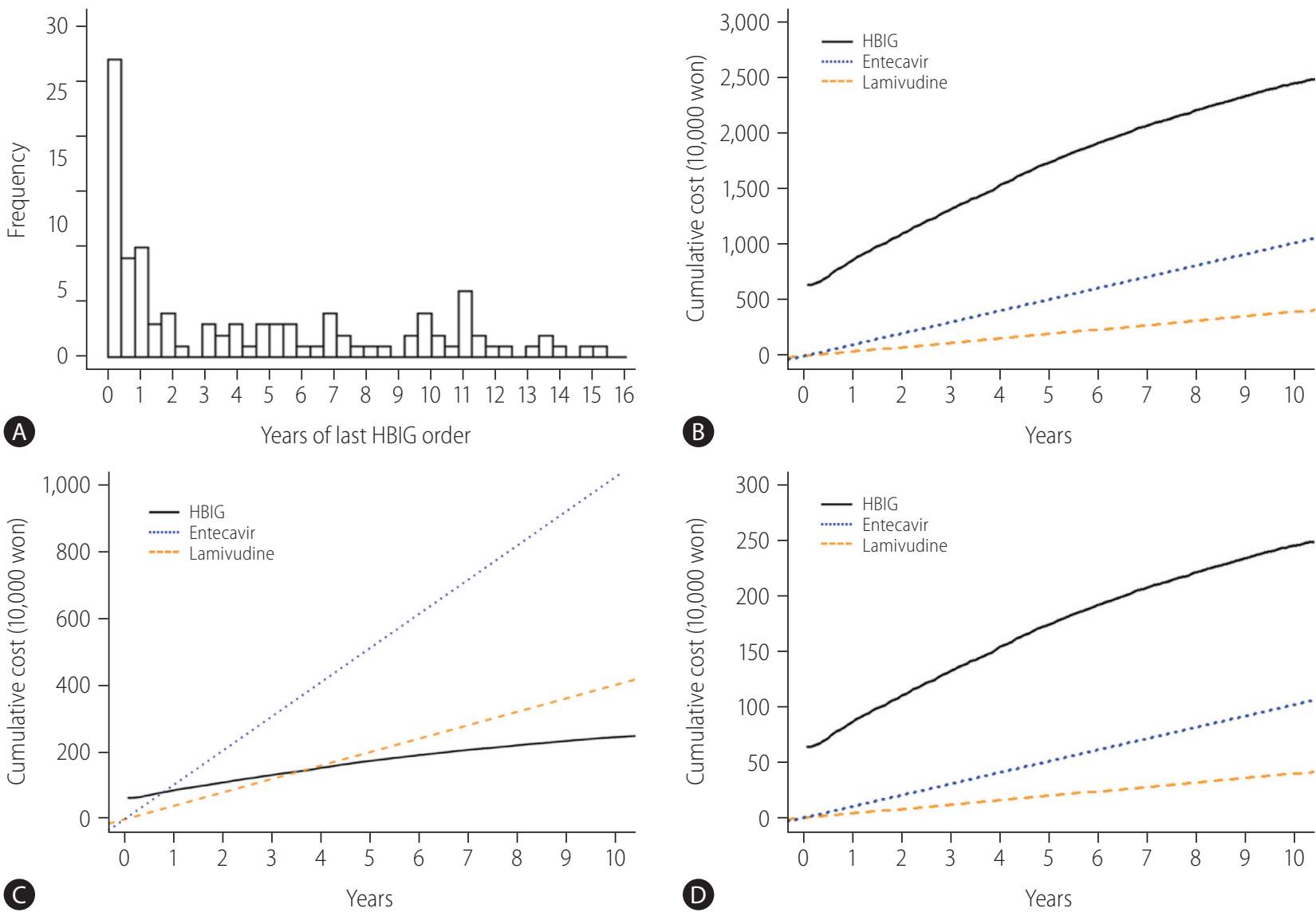

Figure 4. Distributions of the year of last HBIG order and analysis of cumulative cost between HBIG and NAs in non-HBV recipients with anti-HBC positive donors. (A) Distribution of the year of last HBIG order in non-HBV recipients with anti-HBc positive donors. (B) Analysis of cumulative cost between $\mathrm{HBIG}$ and NAs without insurance in non-HBV recipients with anti-HBc positive donors. (C) Analysis of cumulative cost to be paid by patient between $\mathrm{HBIG}$ and NAs when only HBIG is covered by insurance in non-HBV recipients with anti-HBC positive donors. (D) Analysis of cumulative cost to be paid by patient between $\mathrm{HBIG}$ and NAs when both are covered by insurance in non-HBV recipients with anti-HBc positive donors. $\mathrm{HBIG}$, hepatitis B immunoglobulin; anti-HBC, hepatitis B core antibody; NAs, nucleos(t)ide analogues; HBV, hepatitis B virus. 
compared with using NAs continuously (Fig. 4D).

\section{DISCUSSION}

In this study, we found that the anti-HBc status of the liver donor did not affect recipient patient survival or graft survival, even in high MELD ( $\geq 35$ ) patients. The anti-HBc status of the donor did not affect survival or HCC recurrence-free survival in patients who underwent LT due to HCC. The rate of DNH was low in HBsAgnegative recipients receiving anti-HBc-positive grafts when $\mathrm{HBIG}$ monotherapy was used as prophylaxis and anti-HBs titers were maintained above $200 \mathrm{IU} / \mathrm{L}$. However, the DNH rate was high in both anti-HBs/anti-HBc negative recipients. In addition, when $\mathrm{HBIG}$ was used as prophylaxis, the cumulative cost was higher compared to using NAs continuously.

DNH occurrence rates were very high, 43-94\%, when using anti-HBc-positive grafts before adequate prophylaxis options were available. ${ }^{3}$ During this period, Dickson et al. ${ }^{2}$ reported that a significantly lower 4-year patient survival rate for recipients who received livers from anti-HBc-positive donors than for recipients who received livers from anti-HBc-negative donors. In addition, the mortality rate of recipients who received grafts from anti-HBCpositive donors was 2.4 times higher. However, Prieto et al. ${ }^{15}$ reported that the 4-year patient survival rates were similar between these two groups. In a 2001 survey carried out in the United States, almost half of liver transplant physicians reported that they did not use anti-HBc-positive donors for both anti-HBs and anti$\mathrm{HBC}$ negative patients. ${ }^{16}$ After prophylaxis, it remained unclear whether the donor's anti-HBc positivity affected patient survival. Brandl et al. ${ }^{17}$ reported that 10 -year patient and graft survival rates were significantly lower for recipients receiving anti-HBc grafts, and that anti-HBc-positive organs were associated with decreased graft survival in multivariable analysis. However, in this study, due to the long study period of 36 years, there were many changes in treatment methods such as the use of immunosuppressive agents, and a great deal of clinician experience accumulated, leading to selection bias. In addition, there was a mixture of groups that did or did not receive antiviral prophylaxis. On the other hand, several other studies reported that anti-HBc positivity of donors did not affect patient or graft survival. ${ }^{12,18-20}$ MacConmara et al. ${ }^{18}$ and Lei et al. ${ }^{12}$ reported that 5-year patient and graft survival rates were similar between recipients with anti-HBc-positive grafts and anti-HBc-negative grafts. Wong et al. ${ }^{20}$ analyzed long-term outcomes and found that the 10-year patient and graft survival rates were similar between recipients with anti-HBc-positive grafts and anti-HBc-negative grafts, even among high MELD $(\geq 35)$ patients. In addition, they reported that the anti-HBc positivity of donors did not affect 10-year patient survival and disease-free survival rates in HCC patients. Similarly, in our study, there were no significant differences between the two groups in the 10 -year patient survival rates $(67.7 \%$ vs. $70.3 \%, P=0.113)$, whereas the graft survival rates were significantly different between the two groups. However, anti-HBc positivity of donors did not affect patient or graft survival rates in multivariable analysis. In addition, anti-HBc positivity of donors did not affect patient or graft survival in high MELD score patients, and had no effect on the patient survival or disease-free survival in HCC patients. Although anti-HBc-positive grafts are considered suboptimal, patient and graft survival may no longer be affected by DNH with adequate prophylaxis, but rather by graft quality. In our study, although donors in the anti-HBc-positive group were older and had higher BMIs, the patient and graft survival were not inferior to those in the anti-HBc-negative group. Therefore, in HBV endemic areas such as Korea, anti-HBc-positive grafts may be used with less hesitance.

There are three strategies for preventing DNH when using antiHBc-positive grafts: use of HBIG alone, NAs alone, or a combination of HBIG and NAs. According to current international guidelines, lamivudine monotherapy is recommended as the most costeffective choice. ${ }^{6,7}$ However, Wong et al. ${ }^{20}$ reported that all cases of DNH occurred in patients who received lamivudine, whereas no cases of DNH occurred in patients who received entecavir in their large cohort. In a 2010 systematic review, DNH occurred in 27\% of both anti-HBs/anti-HBc negative recipients with HBIG monoprophylaxis compared to $3.4 \%$ of both anti-HBs/anti-HBc negative recipients with lamivudine monoprophylaxis. ${ }^{21}$ A recent systematic review and meta-analysis also showed that DNH occurred in $23 \%$ of anti-HBs-negative patients with HBIG monoprophylaxis compared to $1.0 \%$ of anti-HBs-negative patients with NAs monoprophylaxis. ${ }^{8}$ Saab et al. ${ }^{22}$ reported that the DNH rate was $2.7 \%$ with lamivudine prophylaxis compared to $3.6 \%$ with a combination of $\mathrm{HBIG}$ and lamivudine, and that the cost for the first year of combination therapy was about 20 times higher than that of lamivudine alone. Thus, HBIG is not recommended for preventing DNH in current guidelines. ${ }^{6,7}$ However, in Korea, the use of HBIG and NAs to prevent HBV recurrence when HBsAg-positive patients undergo LT is covered under Korean national health insurance, but when HBsAg-negative patients receive anti-HBc-positive grafts, the use of NAs is not covered and only the use of HBIG is covered by nation- 
al health insurance. Thus, HBIG monoprophylaxis is the standard strategy for HBsAg-negative patients who receive anti-HBc-positive grafts in our institution. The overall incidence of DNH was $0.9 \%(1 / 117)$ in our cohort. This is a very low incidence compared to previous studies. The incidence of DNH was $9.1 \%$ (1/11) in both anti-HBs/anti-HBC negative recipients, but the sample size was small. In addition, even though the anti-HBs titer in the patient with DNH development fell below $200 \mathrm{IU} / \mathrm{L}$, the delay in HBIG administration because we missed it for 4 months seems to have affected DNH.

Wright et al. ${ }^{23}$ evaluated the cost-effectiveness of entecavir compared to lamivudine for prophylaxis in anti-HBc-positive livers. They reported that, in the United States, lamivudine was considered the optimal strategy for recipients receiving anti-HBc-positive liver grafts because entecavir was about three times more expensive to use than lamivudine, and the effect was not significantly different. In our study, the anti-HBs titer was maintained above $200 \mathrm{IU} / \mathrm{L}$ within 2 years, and about half of the recipients discontinued HBIG administration. However, when comparing the cumulative cost at the end of 5 years, using HBIG costs about three times more than using entecavir and nine times more than using lamivudine. Using HBIG as a prophylaxis for recipients receiving anti-HBc-positive liver has a low DNH incidence of $0.9 \%$ compared to other studies. Considering that in the Korean insurance system only the use of HBIG is covered, this treatment seems to be superior in terms of cost effectiveness in Korean patients in this cohort.

There are several limitations to this study. This is a retrospective study with a single-center cohort, and the duration of this cohort (18 years) is long. Although we used anti-HBc-positive grafts with standardized HBV prophylaxis in this period, selection biases inevitably occurred because new treatment methods, such as immunosuppression protocols, were developed during the study period and the experience of the clinicians increased. Since Korea is an HBV endemic area, most patients who were negative for hepatitis B surface antibody were previously vaccinated with $\mathrm{HBV}$, which may have contributed to lowering the DNH rate. Since 2018, the Korean insurance system started to cover NAs as well as HBIG. The use of HBIG may no longer have a definite cost advantage, but the difference in total cost paid by patients is not great, so the use of HBIG may be worth considering.

In conclusion, the use of anti-HBc-positive grafts in and of itself does not affect patient survival or graft survival. HBIG monoprophylaxis has good outcomes for preventing DNH, and the patient's long term cost burden can be low in Korea considering the insurance system in this cohort.

\section{Authors' contribution}

Kyeong Deok Kim: acquired data, analyzed and interpreted data, and wrote the manuscript; Ji Eun Lee: analyzed and interpreted data, and wrote the manuscript; Jong Man Kim: designed the project, analyzed and interpreted data, and wrote the manuscript; Na Young Hwang and Kyunga Kim: analyzed and interpreted data; Okjoo Lee, Jinsoo Rhu, and Gyu-Seong Choi: acquired data; Jae-Won Joh: interpreted data.

\section{Conflicts of Interest}

The authors have no conflicts to disclose.

\section{SUPPLEMENTARY MATERIAL}

Supplementary material is available at Clinical and Molecular Hepatology website (http://www.e-cmh.org).

\section{REFERENCES}

1. Chazouillères O, Mamish D, Kim M, Carey K, Ferrell L, Roberts JP, et al. "Occult" hepatitis $B$ virus as source of infection in liver transplant recipients. Lancet 1994;343:142-146.

2. Dickson RC, Everhart JE, Lake JR, Wei Y, Seaberg EC, Wiesner RH, et al. Transmission of hepatitis $B$ by transplantation of livers from donors positive for antibody to hepatitis B core antigen. The National Institute of Diabetes and Digestive and Kidney Diseases Liver Transplantation Database. Gastroenterology 1997;113:1668-1674.

3. Takemura N, Sugawara Y, Tamura S, Makuuchi M. Liver transplantation using hepatitis $B$ core antibody-positive grafts: review and university of Tokyo experience. Dig Dis Sci 2007;52:2472-2477.

4. Korean Association for the Study of the Liver (KASL). KASL clinical practice guidelines for management of chronic hepatitis B. Clin Mol Hepatol 2019;25:93-159.

5. Lee KW, Lee DS, Lee HH, Kim SJ, Joh JW, Seo JM, et al. Prevention of de novo hepatitis B infection from HbcAb-positive donors in living donor liver transplantation. Transplant Proc 2004;36:2311-2312.

6. Huprikar S, Danziger-Isakov L, Ahn J, Naugler S, Blumberg E, Avery RK, et al. Solid organ transplantation from hepatitis B virus-positive donors: consensus guidelines for recipient management. Am J Transplant 2015;15:1162-1172.

7. European Association for the Study of the Liver. EASL clinical practice guidelines: liver transplantation. J Hepatol 2016;64:433-485. 
8. Yang Y, Huang A, Zhao Y. Effect of hepatitis B surface antibody in patients with core antibody-positive liver transplantation: a systematic review and meta-analysis. Hepatol Int 2020;14:202-211.

9. Lee S, Kim JM, Choi GS, Park JB, Kwon CH, Choe YH, et al. De novo hepatitis $b$ prophylaxis with hepatitis $B$ virus vaccine and hepatitis $B$ immunoglobulin in pediatric recipients of core antibody-positive livers. Liver Transpl 2016;22:247-251.

10. Donataccio D, Roggen F, De Reyck C, Verbaandert C, Bodeus M, Lerut J. Use of anti-HBC positive allografts in adult liver transplantation: toward a safer way to expand the donor pool. Transpl Int 2006;19:38-43.

11. Roque-Afonso AM, Feray C, Samuel D, Simoneau D, Roche B, Emile $J F$, et al. Antibodies to hepatitis $B$ surface antigen prevent viral reactivation in recipients of liver grafts from anti-HBC positive donors. Gut 2002;50:95-99.

12. Lei M, Yan LN, Yang JY, Wen TF, Li B, Wang WT, et al. Safety of hepatitis $B$ virus core antibody-positive grafts in liver transplantation: a single-center experience in China. World J Gastroenterol 2018;24:5525-5536.

13. Yamashiki N, Yoshizawa A, Ueda Y, Kaido T, Okajima H, Marusawa $H$, et al. The use of hepatitis $B$ immunoglobulin with or without hepatitis $B$ vaccine to prevent de novo hepatitis $B$ in pediatric recipients of anti-HBc-positive livers. Pediatr Transplant 2018;22:e13227.

14. Mazzaferro V, Regalia E, Doci R, Andreola S, Pulvirenti A, Bozzetti F, et al. Liver transplantation for the treatment of small hepatocellular carcinomas in patients with cirrhosis. N Engl J Med 1996;334:693699.

15. Prieto $M$, Gómez MD, Berenguer $M$, Córdoba J, Rayón JM, Pastor $M$, et al. De novo hepatitis $B$ after liver transplantation from hepatitis $B$ core antibody-positive donors in an area with high prevalence of
anti-HBC positivity in the donor population. Liver Transpl 2001;7:5158.

16. Burton JR Jr, Shaw-Stiffel TA. Use of hepatitis B core antibodypositive donors in recipients without evidence of hepatitis B infection: a survey of current practice in the United States. Liver Transpl 2003;9:837-842.

17. Brandl A, Stolzlechner P, Eschertzhuber S, Aigner F, Weiss S, Vogel W, et al. Inferior graft survival of hepatitis $B$ core positive grafts is not influenced by post-transplant hepatitis B infection in liver recipients-5-year single-center experience. Transpl Int 2016;29:471-482.

18. MacConmara MP, Vachharajani N, Wellen JR, Anderson CD, Lowell JA, Shenoy $S$, et al. Utilization of hepatitis B core antibody-positive donor liver grafts. HPB (Oxford) 2012;14:42-48.

19. Han JH, Kim DG, Na GH, Kim EY, Lee SH, Hong TH, et al. De novo hepatitis $\mathrm{B}$ virus infection developing after liver transplantation using a graft positive for hepatitis B core antibody. Ann Surg Treat Res 2015;89:145-150.

20. Wong TC, Fung JY, Cui TY, Lam AH, Dai JW, Chan AC, et al. Liver transplantation using hepatitis $\mathrm{B}$ core positive grafts with antiviral monotherapy prophylaxis. J Hepatol 2019;70:1114-1122.

21. Cholongitas E, Papatheodoridis GV, Burroughs AK. Liver grafts from anti-hepatitis B core positive donors: a systematic review. J Hepatol 2010;52:272-279.

22. Saab S, Waterman B, Chi AC, Tong MJ. Comparison of different immunoprophylaxis regimens after liver transplantation with hepatitis B core antibody-positive donors: a systematic review. Liver Transpl 2010;16:300-307.

23. Wright AJ, Fishman JA, Chung RT. Lamivudine compared with newer antivirals for prophylaxis of hepatitis $B$ core antibody positive livers: a cost-effectiveness analysis. Am J Transplant 2014;14:629-634. 